\title{
OB STARS IN THE SOLAR NEIGHBORHOOD. II. KINEMATICS
}

\author{
F. ELIAS \\ Facultad de Física, Departamento de Física Atómica, Molecular, y Nuclear, Universidad de Sevilla, Apartado 1065, 41080 Sevilla, Spain \\ E. J. Alfaro \\ Instituto de Astrofísica de Andalucía, CSIC, Apartado 3004, 18080 Granada, Spain \\ AND \\ J. CABRERA-CAÑO \\ Facultad de Física, Departamento de Física Atómica, Molecular, y Nuclear, Universidad de Sevilla, Apartado 1065, 41080 Sevilla, Spain; and \\ Instituto de Astrofísica de Andalucía, CSIC, Apartado 3004, 18080 Granada, Spain \\ Received 2006 February 24; accepted 2006 May 17
}

\begin{abstract}
Using the spatial classification method and the structural parameters estimated for the Gould Belt (GB) and the local Galactic disk (LGD) from a previous paper, we have evaluated spatial membership probabilities for a sample of $\mathrm{O}$ and B stars from the Hipparcos catalog (Perryman and coworkers) with available proper motions and radial velocity data. Thus, being able to study the space velocity fields of both systems, we conclude that the GB and the LGD present different statistical distributions, both in velocity space and in phase space. In light of their possible existence as distinct systems, we analyze different kinematic aspects in the vicinity of the Sun, and we find the GB to be responsible for the highly negative vertex deviation found for the OB stars in the solar neighborhood. We also find that the GB noticeably alters the estimation of the Oort constants in the Galactic disk; thus, in order to establish comparisons with other kinematic studies based on older stellar populations, a careful pruning of the GB members must be performed. Further analysis of the GB velocity field and the moving groups that contribute to it suggest the possibility that the GB can be the result of a chance superposition of moving groups. We propose for future investigations the dynamical analysis of these moving groups in order to probe the origin of the GB.
\end{abstract}

Key words: solar neighborhood — stars: early-type — stars: kinematics

\section{INTRODUCTION}

Since the discovery of the Gould Belt (GB) as a system of bright stars forming an angle of about $20^{\circ}$ with the Galactic disk (Gould 1879; Herschel 1847), many efforts have been devoted to unraveling its complex structure of stars and the associated interstellar medium. When studies of its kinematics began to appear in the second half of the past century, the peculiarities of its behavior made the global picture of the GB even more puzzling.

The most striking discovery was the expansion of the stellar component (Bonneau 1964; Lesh 1968; Fricke \& Tsioumis 1975; Frogel \& Stothers 1977; Westin 1985; Comerón et al. 1994), which cannot be satisfactorily explained by a single explosive event (Lesh 1968; Moreno et al. 1999), making it difficult to trace back to the origin of the GB by reversing this movement. Models considering Galactic density waves generated by perturbations from the spiral arms cannot account for this expansion either (Westin 1985; Comerón \& Torra 1991). Also, the shell of gas associated with the GB presents evidence of expansion according to the works of Lindblad (1967), Olano (1982), Elmegreen (1982), and Moreno et al. (1999).

Because the age of the GB (between 20 and 90 Myr; see Torra et al. 2000 for a detailed discussion) is a considerable fraction of the period of vertical oscillation of the stars over the Galactic plane under the influence of the gravitational potential of the Galaxy, the spatial coherence of the GB must correspond to a kinematical coherence that prevents the dissolution of the structure into the Galactic plane. Comerón (1999) and Perrot \& Grenier (2003) find a global oscillation of the GB around an axis different from the spatial line of nodes where the GB and the Galactic plane cross each other.

All this leads Elmegreen et al. (2000) to include the GB as part of the hierarchy of stellar complexes within the Milky Way, as a second-level structure subordinated to the local arm (the OrionCygnus spur). Thus, the GB would be the star formation complex closest to us, so the study of its global properties will help shed light on the possible origin and evolution of these complexes.

In a previous article (Elias et al. 2006, hereafter Paper I) we had developed a three-dimensional spatial classification method to separate the GB stars from the local Galactic disk (LGD) stars. Having obtained the structural parameters of both systems exclusively through spatial considerations, we now classify a sample of Hipparcos (Perryman et al. 1997) OB stars with space velocities, in order to compare the kinematics of both structures.

Thus, in $\S 2$ we first build a sample of O-B6 Hipparcos stars with proper motions and radial velocity data that we then analyze in order to study the kinematic properties of the young Galactic disk. We begin $(\S 3)$ with the identification of the moving groups in the velocity field, and then $(\S 3.1)$ we separate the GB from the LGD stars, choosing a membership to either system for the detected moving groups. The next step to enhance our analysis of the young disk velocity field is the elimination of the systematic effects on the velocities of solar motion and Galactic differential rotation. We obtain the residual velocities, the analysis of which also yields different kinematic behaviors for the GB and the LGD $(\S 4)$. The study of the velocity ellipsoids confirms such a difference ( $\S 4.1)$. The effect of the GB on the determination of the Oort constants is addressed in $\S 5$. Finally, some conclusions are given in $\S 6$.

\section{STAR SAMPLE}

We select a sample of 1156 stars of spectral types O-B6 and luminosity classes III, IV, and V from the Hipparcos cata$\log$ (Perryman et al. 1997). Photometric data from the Hauck \& 
Mermilliod (1998) catalog, as well as radial velocity data from the Barbier-Brossat \& Figon (2000) and Grenier et al. (1999) catalogs, have been added when available. Thus, the compilation includes

1. HIP, the Hipparcos identifier number.

2. Spectral type.

3. $V$, the Johnson visual magnitude.

4. Trigonometric parallax (milliarcseconds).

5. Standard error in trigonometric parallax (milliarcseconds).

6. Right ascension for the epoch J1991.5 in the International Celestial Reference System (ICRS) (degrees).

7. Declination for the epoch J1991.5 in the ICRS (degrees).

8. Proper motion in right ascension, $\mu_{\alpha} \cos \delta\left(\operatorname{mas~} \mathrm{yr}^{-1}\right)$.

9. Standard error in proper motion in right ascension $\left(\right.$ mas $\left.^{-1} r^{-1}\right)$.

10. Proper motion in declination, $\mu_{\delta}\left(\right.$ mas $\left.\mathrm{yr}^{-1}\right)$.

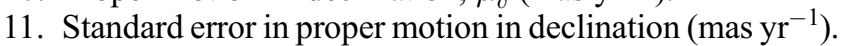

12. Radial velocity $\left(\mathrm{km} \mathrm{s}^{-1}\right)$ from Barbier-Brossat \& Figon (2000).

13. Quality of radial velocity from Barbier-Brossat \& Figon (2000).

14. Radial velocity $\left(\mathrm{km} \mathrm{s}^{-1}\right)$ from Grenier et al. (1999).

15. Error in radial velocity $\left(\mathrm{km} \mathrm{s}^{-1}\right)$ from Grenier et al. (1999).

16. The $u v b y \beta$ photometry data from Hauck \& Mermilliod (1998).

For the distance estimation we have used Hipparcos trigonometric parallaxes only if the relative error is lower than or equal to $10 \%$. Otherwise, uvby $\beta$ Strömgren photometry has been used to estimate the distance through the Balona \& Shobbrook (1984) $M_{V}(\beta)$ calibration. If no data were available, spectrophotometric distances from the apparent visual magnitude $V$ and the SchmidtKaler (1982) calibration for spectral types were chosen. We have compared the three different distance estimations, looking for any possible systematic biases among them. With this purpose, we have selected 950 stars from the initial catalog with Strömgren photometry data.

Several authors (Arenou \& Luri 1999; Maíz-Apellániz 2001, 2005; Schröder et al. 2004), studying the distance calibration comparisons with the distances obtained from trigonometric parallaxes, have dealt with the problem of analyzing the biases that the sample selection effects introduce. The estimation of these biases is very complex because it depends, among other variables, on the spatial distribution of the sample. Maíz-Apellániz (2005) demonstrates that the real distance probability distribution for individual stars will always be ill-behaved when that distance tends to infinity and a constant underlying spatial distribution has been assumed for the sample. This idea had already been suggested by Arenou \& Luri (1999), who proposed that - in order to avoid any truncation biases - for comparison purposes one should use a sample not selected by any limit in the relative error of the trigonometric parallax, including the negative parallaxes. We use this methodology for the comparison between trigonometric, photometric, and spectroscopic parallaxes, using the complete sample with spectral types up to B6.

The results of the analysis demonstrate that the Strömgren photometric parallaxes $\left(\pi_{\text {Str }}\right)$ are very similar to the Hipparcos trigonometric parallaxes, as Kaltcheva \& Knude (1998) had already demonstrated, but the former present, in comparison with the spectroscopic parallaxes $\left(\pi_{\mathrm{SK}}\right.$, derived from the Schmidt-Kaler calibration), a functional relationship in the form of $\pi_{\mathrm{Str}} / \pi_{\mathrm{SK}} \sim 1.21$. Nevertheless, a recent study of O stars (Maíz-Apellániz 2005) demonstrates that the Hipparcos trigonometric parallaxes and the spectroscopic parallaxes are very similar for this spectral type. Where does this difference that we find in their values come from? We have to consider that our spectroscopic calibration is based on three steps: (1) spectral classification, (2) calibration of the absolute magnitude for each spectral type; and (3) evaluation of the reddening from the intrinsic color values.

The spectral classification of the Hipparcos catalog comes from different sources and thus is far from being uniform. On the other hand, the catalog of O stars (Maíz-Apellániz et al. 2004) used by Maíz-Apellániz (2005) for the comparison of the parallaxes was derived from a group of homogeneous and precise stellar spectra. Thus, it must not be inferred from our result that the Schmidt-Kaler calibration has systematic biases but that the spectroscopic parallaxes of the O-B6 stars from the Hipparcos catalog - according to the spectral classification within the catalog-show a systematic error when we compare them with the Strömgren photometric parallaxes (which, as we said, show no significative differences from the Hipparcos trigonometric parallaxes).

In this work we do not intend to perform an exhaustive study of the problems that the diverse methodologies of obtaining distance calibrations produce. We have only unified for the purpose of this work the different distance calibrations used for our sample. To do this, we have tied the spectroscopic parallaxes to those derived from Strömgren photometry, applying a correction of $21 \%$ to the former. We want to stress that the comparison between the different distance calibrations has been made strictly through their respective parallaxes.

We have also chosen the Barbier-Brossat \& Figon (2000) radial velocity and quality data when available; otherwise, radial velocities and errors from Grenier et al. (1999) have been used. Finally, a distance limit of $1 \mathrm{kpc}$ has been imposed, thus reducing the sample to 881 stars.

While the Hipparcos catalog is complete down to $V \sim 7.9$, and to $V \sim 7.5$ for O-B6 stars, in Figure 1 we can see from a histogram that our sample is complete only down to a magnitude of $V \sim 6.5$. This is caused by the necessity of having radial velocity data available for the stars in our sample, as is also observed in Fernández (2005); in that work, the completeness of the sample of $\mathrm{O}$ and B stars from the Hipparcos catalog falls down from $V \sim 7.9$ to $\sim 6$ when the stars without radial velocity data are removed.

\section{IDENTIFICATION OF MOVING GROUPS IN THE SAMPLE AND THEIR MEMBERSHIP}

We have calculated the space velocities from the proper motions and radial velocities (Johnson \& Soderblom 1987) for the stars in our sample. Their density field is represented in the three contour plots of Figure 2. We note in this image that the velocity field is dominated by several maxima that may correspond to associations of stars (not necessarily bound) with a small velocity dispersion, known as moving groups (e.g., Proctor 1869; Eggen 1963).

The first and most prominent maximum, located around $(U$, $V, W)=(-6.5,-19,-7) \mathrm{km} \mathrm{s}^{-1}$, is certainly associated with the Pleiades moving group. The exact situation of the peak may differ slightly from the estimation given by other authors, but we must consider that the moving group always appears as a maximum in the velocity space with a certain width. For instance, Chen et al. (1997), working with a sample of B, A, and F stars from the Hipparcos Input Catalogue (Turon et al. 1992) and uvby $\beta$ photometry, find that the maximum is located at $(U, V, W)=(-10$, $-19,-8.1) \mathrm{km} \mathrm{s}^{-1}$, the standard deviations for the three components being, respectively, $7.9,8.6$, and $5.8 \mathrm{~km} \mathrm{~s}^{-1}$.

The second most prominent peak in Figure 2 is located around $(U, V, W)=(-17,-11,-5) \mathrm{km} \mathrm{s}^{-1}$. We have identified it as the moving group related to the supercluster IC 2391. Its position 


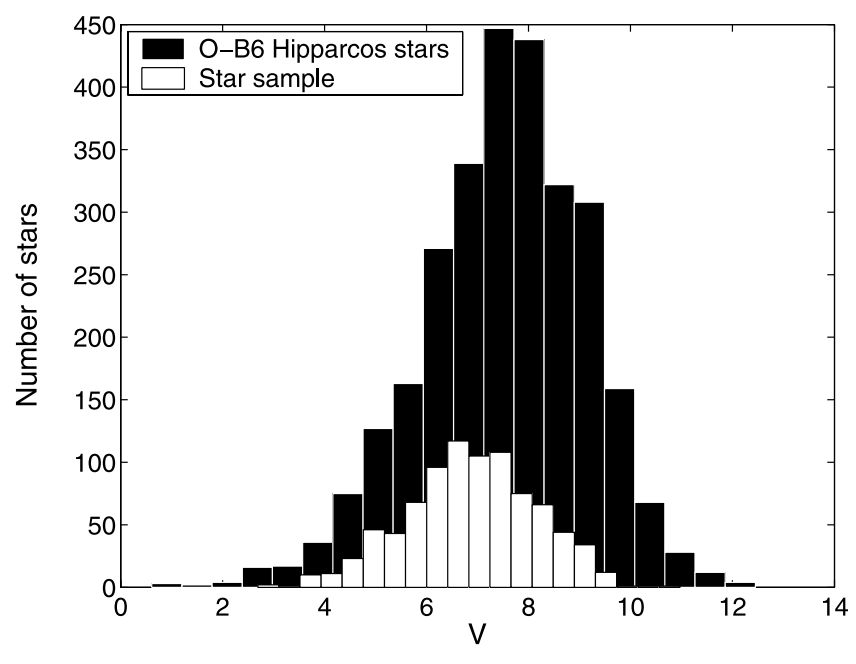

FIG. 1.- Histograms of the $V$ magnitude in the Johnson system for the O-B6 stars in the Hipparcos catalog (Perryman et al. 1997) and for our star sample.

in velocity space is estimated by Chen et al. (1997) at $(U, V, W)=$ $(-15.9,-13.1,-4.5) \mathrm{km} \mathrm{s}^{-1}$, with the standard deviation being $\left(\sigma_{U}, \sigma_{V}, \sigma_{W}\right)=(4.1,6.2,3.0) \mathrm{km} \mathrm{s}^{-1}$.

The third maximum, centered around $(U, V)=(-11,-8.5)$ $\mathrm{km} \mathrm{s}^{-1}$, is more diffuse and difficult to identify. We have decided to follow the criteria of Asiain et al. (1999), who, based on the studies by Comerón (1992), rule out the possibility of linking this region in velocity space with the Coma Berenices cluster, in favor of a probable bond with the Cassiopeia-Taurus association, at $(U, V)=(-9.9,-6.1) \mathrm{km} \mathrm{s}^{-1}$. This association has an age between $50 \mathrm{Myr}$, as the probable expansion age estimated by Blaauw (1956) from a sample of stars of spectral type B5 or earlier, and $90 \pm 10 \mathrm{Myr}$, as determined from the lithium depletion boundary by Stauffer et al. (1999). In the detailed study of the nearby OB associations by de Zeeuw et al. (1999) the authors find a physical relation between the Cas-Tau group and the $\alpha$ Persei cluster. The main-sequence turnoff age for this cluster is about $50 \mathrm{Myr}$ (Meynet et al. 1993), so the age of both Cas-Tau and $\alpha$ Persei could be the same (e.g., Brown 2002). Note that in Paper I we found that $\alpha$ Persei is located well within the spatial boundaries of the GB.

\subsection{Classification of the Sample}

In order to observe how these moving groups contribute to the two systems in study, we classify the sample and determine the membership of the stars in either the GB or the LGD. In Paper I we obtained several estimations of the parameters that characterize both systems in our model. We work with the solution for the full O-B6 sample of Paper I with an exponential model for the stellar distribution in the direction perpendicular to the mean planes.

Thus, using that estimation as the true value of the GB and the LGD mean planes, we simply apply our separation algorithm to our current star sample with kinematic data. No iteration in order to reevaluate the mean planes is performed; we just assign membership probabilities to the stars according to the planes estimated in Paper I. Also, spatial outliers are eliminated according to the procedure explained in that paper; the remaining sample has 776 stars. We obtain a separation between the GB and the LGD based exclusively on the spatial positions of these stars. Yet we can see in Figure 3 how a difference in their velocity fields is obtained as a result.

The most striking difference is that in the $U-V$ projection (Fig. 3 , top panels) the three moving groups that we had found in the full sample (Fig. 2, top) now distinctly belong to either the GB or the
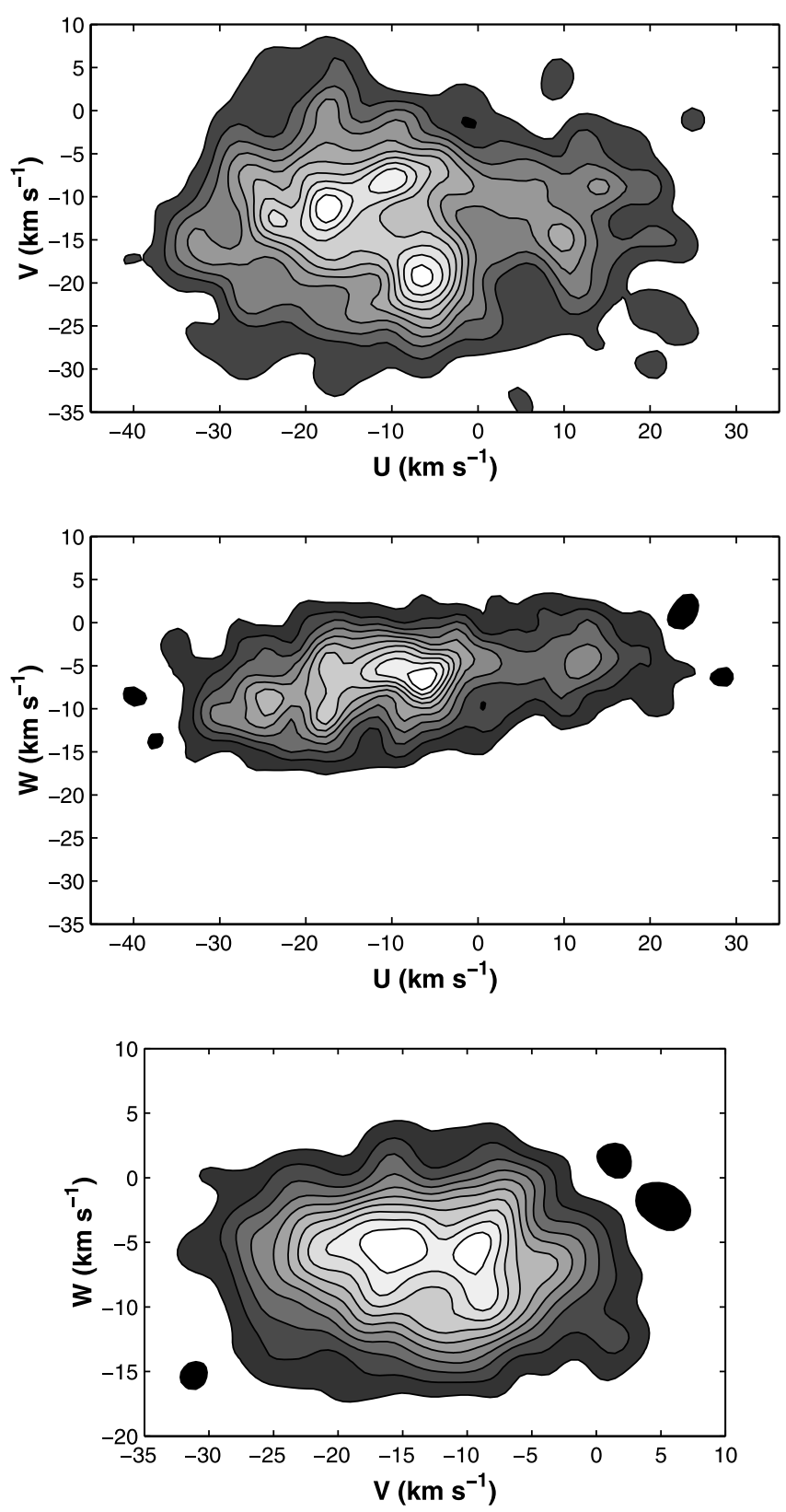

FIG. 2.-Distribution of space velocities for the star sample.

LGD. The two maxima associated with the Pleiades and IC 2391 appear only in the GB velocity field (Fig. 3, top left), while the CasTau peak remains visible only in the LGD field (Fig. 3, top right). This is not surprising, if we consider that the Pleiades moving group is spatially related to the Sco-Cen association, which is one of the main components of the GB (Moreno et al. 1999). Also, we know that IC 2391 is a young cluster, its age being about $30 \mathrm{Myr}$ (Stauffer et al. 1997). In a recent study of tangential velocities, Piskunov et al. (2006) concluded that the kinematic probability of its belonging to the GB is $73 \%$. Note that we have arrived at a similar conclusion by a process based solely on the spatial positions of the stars, and thus independent of the result in the cited paper.

We must also note that a late-type population of young stars has been associated with both the Pleiades and IC 2391 by Montes et al. (2001). In that paper these moving groups are described as centered around $(U, V, W)=(-11.6,-21,-11.4)$ and $(U$, $V, W)=(-20.6,-15.7,-9.1) \mathrm{km} \mathrm{s}^{-1}$, respectively, with a dispersion of about $8 \mathrm{~km} \mathrm{~s}^{-1}$ around the central positions. Not 

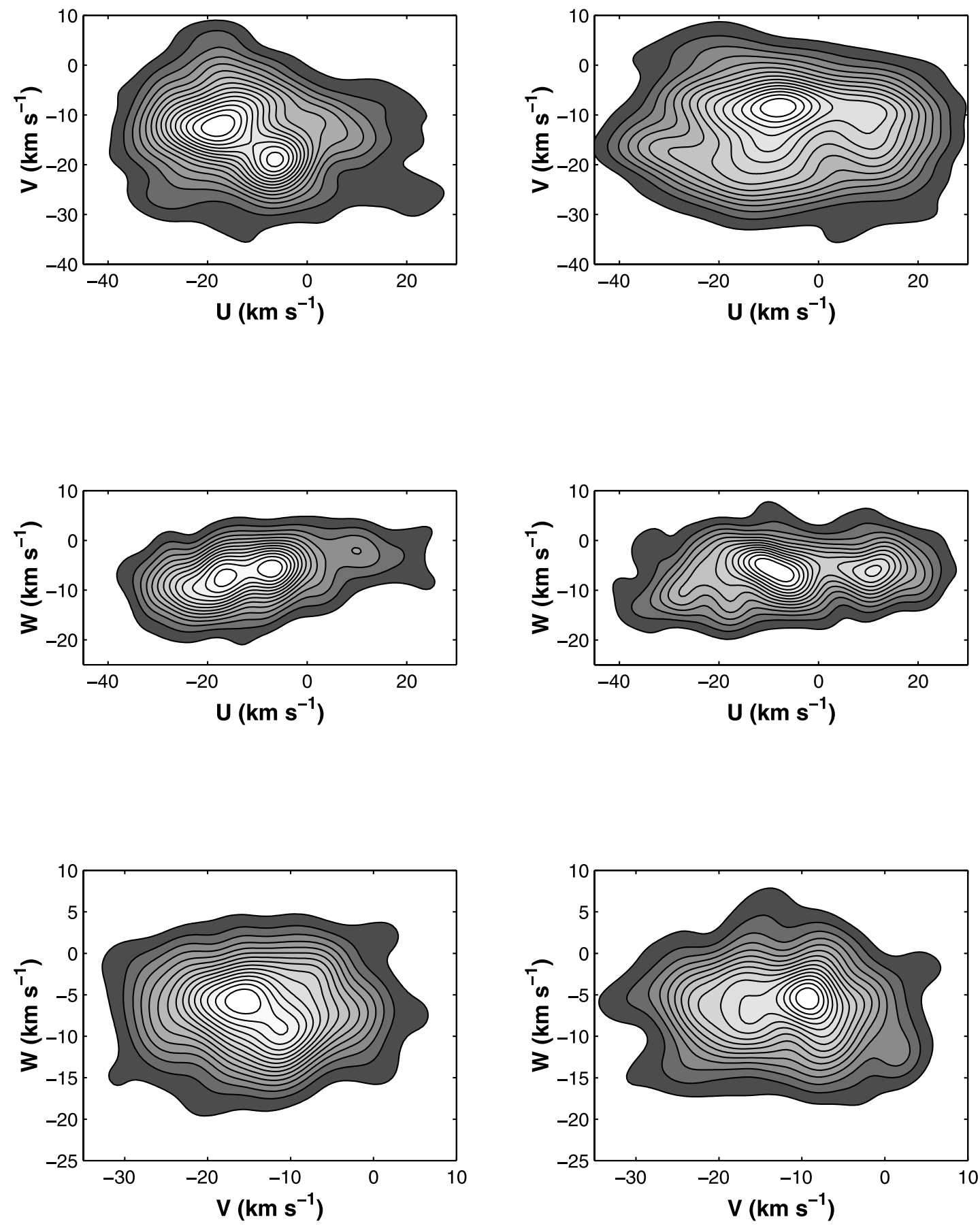

FIG. 3.-Distribution of space velocities for the GB (left panels) and the LGD (right panels) stars. 
surprisingly, a late-type component of stars of about 30-80 Myr of age had already been associated with the GB disk structure by Guillout et al. (1998) when studying the X-ray sources in the ROSAT All-Sky Survey.

Finally, we also observe that in the $U$ - $W$ projection (Fig. 3, middle right) a new maximum clearly rises that has a correspondence with a small protuberance around $U=11 \mathrm{~km} \mathrm{~s}^{-1}$ in the $U-V$ projection (Fig. 3, top right). It was also present, although weak, in the velocity density field of the full sample (Fig. 2, top and middle). We have not found an exact correspondence to this possible moving group among the structures in the solar neighborhood, but the positive value of the $U$-component makes us think that it may be related to the Sirius supercluster (e.g., Eggen 1996; Dehnen 1998; Asiain et al. 1999).

\section{ANALYSIS OF THE RESIDUAL VELOCITIES}

If we want to improve our study of the kinematic differences between the GB and the LGD, we must work with residual velocities. In this way, the systematic effects of the Galactic kinematics do not interfere with our comparison. Thus, we now correct the space velocities of the stars in our sample for solar motion (using the classical estimation of Delhaye [1965]: $\left[U_{\odot}, V_{\odot}, W_{\odot}\right]=$ $\left.[9,12,7] \mathrm{km} \mathrm{s}^{-1}\right)$ and for Galactic differential rotation using the values given by Kerr \& Lynden-Bell (1986).

In order to refine the analysis of the velocity distributions, we must also eliminate outliers of kinematic nature that may be present in the sample. That is, we eliminate the stars located in the regions of very low density in velocity space for our sample under study. We achieve this by running the OUTKER algorithm (CabreraCaño \& Alfaro 1985), which reduces the sample to a final number of 752 stars. We then again separate the sample into GB and LGD members. Now we want to compare both distributions in an $N$-dimensional space, where $N=6$ (phase space) or $N=3$ (velocity space). In order to achieve this, we employ a multidimensional, nonparametric two-sample test: the Cramer test (Baringhaus \& Franz 2004). The test statistic is the difference of the sum of all the Euclidean interpoint distances between the random variables from the two different samples:

$$
\begin{aligned}
T= & \frac{m n}{m+n}\left[\frac{2}{m n} \sum_{i, j}^{m, n} \phi\left(\left\|\boldsymbol{X}_{i}-\boldsymbol{Y}_{j}\right\|^{2}\right)\right. \\
& \left.-\frac{1}{m^{2}} \sum_{i, j=1}^{m} \phi\left(\left\|\boldsymbol{X}_{i}-\boldsymbol{X}_{j}\right\|^{2}\right)-\frac{1}{n^{2}} \sum_{i, j=1}^{n} \phi\left(\left\|\boldsymbol{Y}_{i}-\boldsymbol{Y}_{j}\right\|^{2}\right)\right],
\end{aligned}
$$

where $\boldsymbol{X}_{i}$ and $\boldsymbol{Y}_{i}$ are the point vectors of each sample member, $m$ and $n$ are the respective sizes of the samples, and $\phi$ is a kernel function; for this particular case we have used the Cramer kernel implemented by Franz (2004) in the R statistical environment ( R Development Core Team 2005).

Thus, an analysis of the three-dimensional velocity space $(U$, $V, W$ ) yields that the GB and the LGD distributions are different with a $99 \%$ confidence level. Similarly, the Cramer test for the six dimensions of the phase space $(X, Y, Z, U, V, W)$ rejects the possibility that the GB and the LGD distributions are the same with a confidence of $99 \%$.

Thus, we confirm that the GB and the LGD are two different stellar systems in the sense that they show a clear statistical separation between their distributions in the phase space. It is unavoidable to take into consideration the contribution of the GB when the young disk is under study, because, as we have demonstrated, the velocities of the OB stars in the solar neighborhood are not statistically independent of their spatial positions.

\subsection{Velocity Ellipsoids}

A more intuitive visualization of the differences between the GB and the LGD residual velocity distributions is in the contour density plots of the three different velocity planes (see Fig. 4). Their disparate shapes and orientations already show that the velocity ellipsoids clearly reflect the distinct kinematic behavior of both stellar systems.

We have estimated the main geometric parameters of the velocity ellipsoids for the whole sample and for the GB and the LGD members separately. The results are displayed in Table 1. Two main results arise from this analysis:

1. The vertex deviation for the GB is highly negative, while that for the LGD is positive.

2. There is the suggestion that the third axis of the velocity ellipsoid for the GB is tilted with respect to the Galactic plane.

The estimation of the vertex deviation in the solar neighborhood from different star samples has produced different values depending on the nature of the sample and on the kinematic variables used in the calculation (see Moreno et al. [1999] for a compilation of previous results). In brief, the general conclusion has been that the vertex deviation becomes more negative as the star sample gets younger. In fact, one of the classic estimates based on space velocities for OB stars (Filin 1957) yielded a value close to $l_{v}=$ $-50^{\circ}$ for the Galactic disk. For years, this result has remained a puzzling issue that has been given several and varied explanations. Most of them can be classified into two classical types: nature or nurture, we could say. Some authors claim that these young stars that formed from a molecular cloud show the same kinematics as the parent cloud at the time of the star formation. In this way, the initial velocity and later expansion define the velocity ellipsoid of the present stellar system. Other authors, however, argue that the effects of different singular events (such as passing through a spiral arm) could also be the cause of the peculiar velocity ellipsoid observed in the young stellar component.

What we conclude from our analysis is that the classic problem of the negative vertex deviation for young stars in the solar neighborhood is a consequence of the presence of the GB. If we eliminate the stars that belong to the GB, the remaining sample of only LGD stars presents a positive vertex deviation $\left(l_{v}^{\mathrm{LGD}}=18^{\circ}\right)$.

Moreno et al. (1999), working with a sample of dwarf O-B5.5 star members of the GB, found that the negative vertex deviation $\left(l_{v} \sim-64^{\circ}\right)$ was caused by the Pleiades moving group. Once this group was removed, Moreno et al. obtained a positive vertex deviation $\left(l_{v}=22^{\circ}\right)$ for the remaining stars in the GB. We now demonstrate that the OB stars of the LGD also have a positive vertex deviation, similar to that found by Moreno et al. (1999) for the GB stars after eliminating the ones belonging to the Pleiades moving group. So when we work with samples (of either GB or LGD stars) in which a single moving group clearly dominates over the others, the vertex deviation is positive and close to $l_{v} \sim 20^{\circ}$, which is the value expected from the dynamic equations of the system for this age group. The negative vertex deviation of the GB seems to originate from the relative positions of the centroids of the two moving groups rather than from the distribution of the residual velocities as a whole. We must note that the analysis of the young stars, especially those belonging to the $\mathrm{GB}$, based on the Schwarzschild distribution from which the velocity ellipsoid is tailored, is not the best suited to describe the reality of the system. The velocity field is dominated, as we have seen, by the presence of moving groups, and thus, it is far from the hypothesis of a homogeneous and stationary system. The problem thus lies in that the classical analysis of the velocity ellipsoid is applied to a set of 

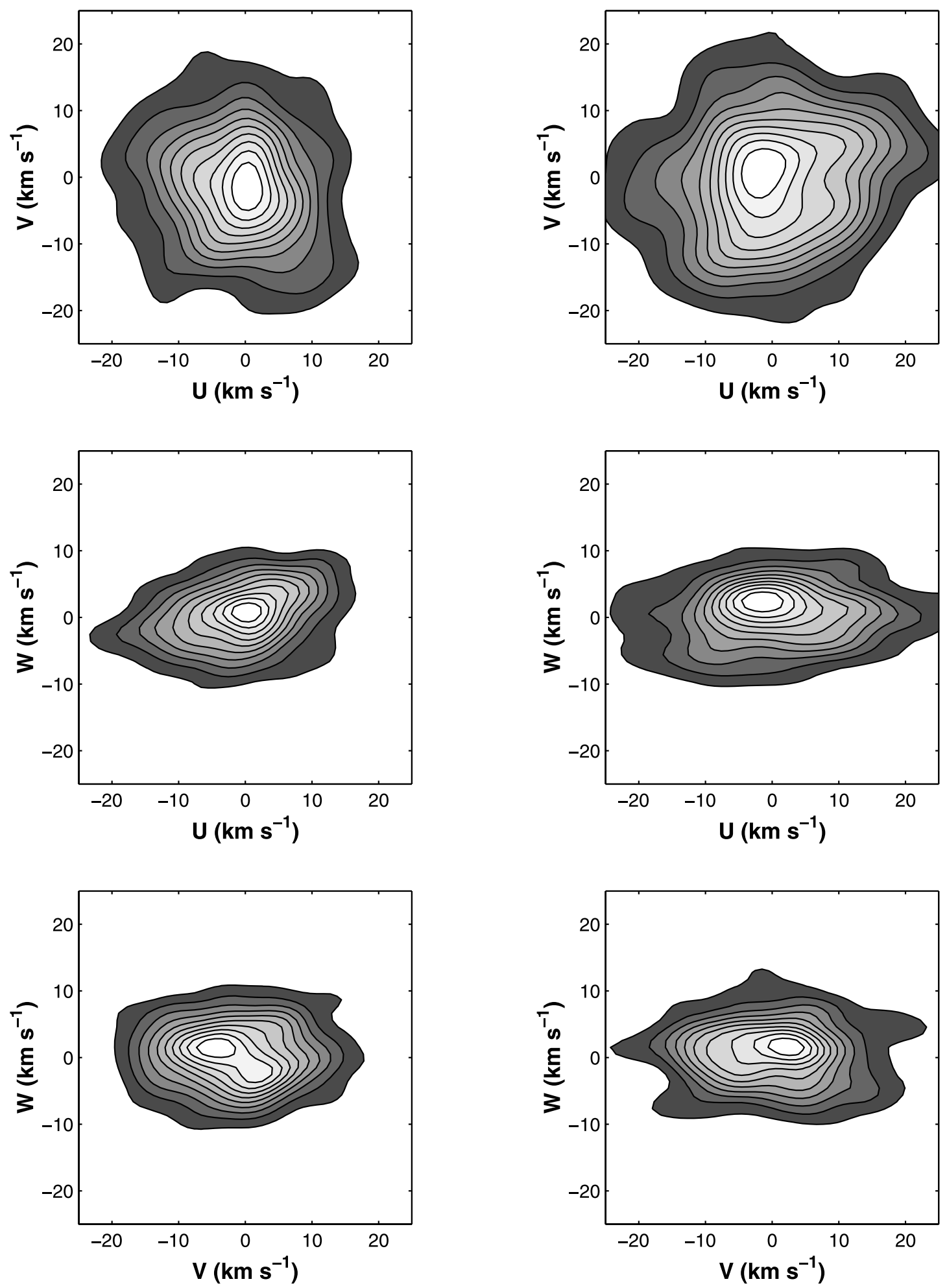

FIG. 4. - Contour density plots of the residual velocities for the GB (left) and the LGD (right) stars. 
TABLE 1

Velocity ElLipsoids

\begin{tabular}{|c|c|c|c|}
\hline Axis & $\begin{array}{c}\sigma \\
\left(\mathrm{km} \mathrm{s}^{-1}\right)\end{array}$ & $\begin{array}{c}l \\
(\mathrm{deg})\end{array}$ & $\begin{array}{c}b \\
(\mathrm{deg})\end{array}$ \\
\hline \multicolumn{4}{|c|}{ Full Sample } \\
\hline$U^{\prime} \ldots \ldots \ldots \ldots \ldots \ldots$ & $10.7 \pm 0.4$ & $-5 \pm 29$ & $6 \pm 4$ \\
\hline$V^{\prime} \ldots \ldots \ldots \ldots \ldots$ & $10.2 \pm 0.4$ & $85 \pm 29$ & $-2 \pm 4$ \\
\hline$W^{\prime} \ldots \ldots \ldots \ldots \ldots \ldots$ & $6.9 \pm 0.6$ & $153 \pm 115$ & $84 \pm 3$ \\
\hline \multicolumn{4}{|c|}{ Gould Belt } \\
\hline$U^{\prime} \ldots \ldots \ldots \ldots \ldots \ldots$ & $9.8 \pm 0.3$ & $-47 \pm 22$ & $-10 \pm 8$ \\
\hline$V^{\prime} \ldots \ldots \ldots \ldots \ldots \ldots \ldots$ & $8.9 \pm 0.3$ & $43 \pm 22$ & $1 \pm 12$ \\
\hline$W^{\prime}$ & $7.1 \pm 0.8$ & $141 \pm 92$ & $80 \pm 12$ \\
\hline \multicolumn{4}{|c|}{ Local Galactic Disk } \\
\hline$U^{\prime} \ldots \ldots \ldots \ldots \ldots \ldots$ & $12.2 \pm 0.6$ & $18 \pm 11$ & $-2 \pm 4$ \\
\hline & $10.2 \pm 0.5$ & $-72 \pm 11$ & $0 \pm 5$ \\
\hline$W^{\prime} \ldots \ldots \ldots \ldots \ldots$ & $7 \pm 1$ & $27 \pm 66$ & $88 \pm 5$ \\
\hline
\end{tabular}

Notes.-The quantities $U^{\prime}, V^{\prime}$, and $W^{\prime}$ represent the principal axes of the ellipsoids, which deviate from the $(U, V, W)$ reference frame. The errors were estimated by bootstrap.

moving groups. Mihalas \& Binney (1981) have already pointed out that the cause of the vertex deviation was the existence of moving groups in the Galactic velocity field. But even though the velocity ellipsoid does not strictly correspond to a physical reality in our case, that does not invalidate our result, that is, the fundamental contribution of the GB to the negative vertex deviation for the $\mathrm{O}$ and B stars.

Thus, the different values of the vertex deviation found in the literature can be explained according to the different proportions of GB stars present in the respective samples. This translates the question about the origin of the negative vertex deviation to the investigation of the origin of the moving groups. Although the latter is out of the scope of this paper, we want to stress that the more we study in detail the nature of the GB, the more we find indications that we must probe both its nature and origin as a set of moving groups.

Another striking result is the inclination of the GB's velocity ellipsoid of about $10^{\circ} \pm 12^{\circ}$ with respect to the $U-V$ plane, although this value does not have a great statistical significance. While the third axis $\left(W^{\prime}\right)$ of the LGD ellipsoid merely shows an inclination of $2^{\circ}$, the GB's ellipsoid inclination $\left(10^{\circ}\right)$ resembles that of the spatial system with respect to the Galactic plane $\left(i_{\mathrm{GB}} \sim\right.$ $14^{\circ}$ as we found in Paper I). Some authors have pointed out that the GB could be oscillating as a whole around the Galactic plane (Comerón 1999; Perrot \& Grenier 2003). But our result does not prevent a different interpretation in terms of a juxtaposition of moving groups: this links back to the idea that a descriptive parameter of the GB, such as the inclination of the minor axis of the ellipsoid, can be interpreted in terms of the relative position (in the velocity space) of two moving groups.

\section{ESTIMATION OF THE OORT CONSTANTS}

Although our sample has not been ideally compiled with the intention of calculating the Oort constants, it is worth performing a basic estimation to obtain information about the GB's contribution to their value. Thus, within the axisymmetric approximation of Oort's model in first order, and including a $K$ expansion term, we solve the condition equations (Smart 1968; Clube 1972; Frogel \& Stothers 1977) for the radial and tangential velocities:

$v_{r}=u_{0} \cos l \cos b+v_{0} \sin l \cos b+w_{0} \sin b+A r \sin 2 l \cos ^{2} b+K$,

$$
\begin{gathered}
v_{l}=-u_{0} \sin l+v_{0} \cos l+A r \cos 2 l \cos b+B r \cos b, \\
v_{b}=-u_{0} \cos l \sin b-v_{0} \sin l \sin b+w_{0} \cos b-A r \sin 2 l \cos b \sin b,
\end{gathered}
$$

where $v_{r}$ is the radial velocity, $v_{l}=4.74057 \mu_{l} r$ and $v_{b}=$ $4.74057 \mu_{b} r$ are the tangential velocities in Galactic longitude $(l)$ and latitude $(b)$, with $\mu_{l}$ and $\mu_{b}$ the respective proper motions and $r$ the heliocentric distance, and $\left(u_{0}, v_{0}, w_{0}\right)=-\left(U_{\odot}, V_{\odot}, W_{\odot}\right)$ are the reflex of the solar motion.

In principle, it would only make sense to estimate the Oort constants for the LGD, eliminating the GB members from the sample. As we have seen from the study of the moving groups and the velocity ellipsoid, the velocity distribution of the GB clearly deviates from the axisymmetric hypothesis; hence, the Oort constants would not correspond to a physical reality in this case. Yet we have solved the equations in order to establish a comparison and thus observe the effects that the presence of the GB introduces in the kinematics of the LGD.

The solutions for the proper motions and for the radial velocity alone are listed in Table 2. The solar motion for the LGD proper motion solution is in good agreement with the IAU standard, $\left(U_{\odot}, V_{\odot}, W_{\odot}\right)=(9,12,7) \mathrm{km} \mathrm{s}^{-1}$ (Kerr \& Lynden-Bell 1986). But the Oort constants differ noticeably from the IAU recommended values of $A=14.4 \pm 1.2 \mathrm{~km} \mathrm{~s}^{-1} \mathrm{kpc}^{-1}$ and $B=$ $-12.0 \pm 2.8 \mathrm{~km} \mathrm{~s}^{-1} \mathrm{kpc}^{-1}$, and instead we have a flat rotation

TABLE 2

\begin{tabular}{|c|c|c|c|c|c|c|}
\hline Sample & $\begin{array}{c}U_{\odot} \\
\left(\mathrm{km} \mathrm{s}^{-1}\right)\end{array}$ & $\begin{array}{c}V_{\odot} \\
\left(\mathrm{km} \mathrm{s}^{-1}\right)\end{array}$ & $\begin{array}{c}W_{\odot} \\
\left(\mathrm{km} \mathrm{s}^{-1}\right)\end{array}$ & $\begin{array}{c}A \\
\left(\mathrm{~km} \mathrm{~s}^{-1} \mathrm{kpc}^{-1}\right)\end{array}$ & $\begin{array}{c}B \\
\left(\mathrm{~km} \mathrm{~s}^{-1} \mathrm{kpc}^{-1}\right)\end{array}$ & $\begin{array}{c}K \\
\left(\mathrm{~km} \mathrm{~s}^{-1}\right)\end{array}$ \\
\hline \multicolumn{7}{|c|}{ Solution from Proper Motions } \\
\hline 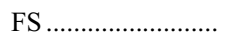 & $9.8 \pm 0.4$ & $13.0 \pm 0.6$ & $6.6 \pm 0.3$ & $14 \pm 1$ & $-18 \pm 1$ & .. \\
\hline 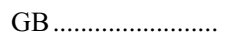 & $9.9 \pm 0.5$ & $13.0 \pm 0.6$ & $6.7 \pm 0.3$ & $11 \pm 2$ & $-20 \pm 1$ & $\ldots$ \\
\hline LGD …….................. & $9.4 \pm 0.8$ & $12.6 \pm 0.9$ & $6.3 \pm 0.3$ & $16 \pm 2$ & $-16 \pm 1$ & $\ldots$ \\
\hline \multicolumn{7}{|c|}{ Solution from Radial Velocity } \\
\hline 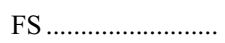 & $8.7 \pm 0.8$ & $15.0 \pm 0.6$ & $11 \pm 2$ & $13 \pm 2$ & $\ldots$ & $-0.6 \pm 0.4$ \\
\hline 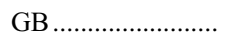 & $10 \pm 2$ & $15 \pm 1$ & $8 \pm 4$ & $9 \pm 2$ & $\ldots$ & $0.4 \pm 0.6$ \\
\hline 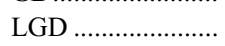 & $9 \pm 1$ & $14 \pm 1$ & $8 \pm 5$ & $16 \pm 3$ & $\ldots$ & $-2 \pm 1$ \\
\hline
\end{tabular}

Oort Constants

Notes.-FS is the full sample. 
curve with $A=-B=16 \mathrm{~km} \mathrm{~s}^{-1} \mathrm{kpc}^{-1}$. Yet the value provided by Kerr \& Lynden-Bell (1986) is a mean over the results obtained by several authors, so it is more revealing to compare our estimation with that of a study similar to ours.

Frogel \& Stothers (1977), working with a sample of O-B5 stars, separating the GB from the LGD, and considering a fixed value of $A=15 \mathrm{~km} \mathrm{~s}^{-1} \mathrm{kpc}^{-1}$, estimated the value of $B$ for both systems, as well as for the whole unclassified sample. For the latter they found a flat rotation curve of $A=-B=15 \mathrm{~km} \mathrm{~s}^{-1} \mathrm{kpc}^{-1}$; for the LGD, $B=-12 \mathrm{~km} \mathrm{~s}^{-1} \mathrm{kpc}^{-1}$; and for the GB, $B=-19 \mathrm{~km}$ $\mathrm{s}^{-1} \mathrm{kpc}^{-1}$, a value similar to our estimation. But regardless of the numerical results, we can also conclude that the global kinematics of the GB differs greatly from the kinematics of the LGD, and that the presence of this stellar system affects the estimation of the parameters describing the kinematics of the solar neighborhood.

On the other hand, the $K$ term is generally lower in absolute value than the estimation by Frogel \& Stothers (1977). We have checked that when solving the equations without this term, the results for the Oort constants do not change significantly. Thus, we do not consider it wise to conclude anything about the possible expansion movements of the system from these results.

The detailed study of the local irregularities in the kinematics of young stars by Torra et al. (2000), from a sample of O and B Hipparcos stars, reveals that for heliocentric distances lower than $600 \mathrm{pc}$ and age groups under $90 \mathrm{Myr}$ (i.e., for a sample with a high proportion of GB members), the value of the Oort constant $B$ is much greater in absolute value than expected for the LGD:

$-13.6 \pm 2.0 \mathrm{~km} \mathrm{~s}^{-1} \mathrm{kpc}^{-1}<B<-20.7 \pm 1.4 \mathrm{~km} \mathrm{~s}^{-1} \mathrm{kpc}^{-1}$.

On the other hand, they find quite a small value for the Oort constant $A$ :

$$
10.5 \pm 2.1 \mathrm{~km} \mathrm{~s}^{-1} \mathrm{kpc}^{-1}<A<5.7 \pm 1.4 \mathrm{~km} \mathrm{~s}^{-1} \mathrm{kpc}^{-1}
$$

Both estimations are perfectly compatible with our result of $(A, B)=(11 \pm 2,-20 \pm 2) \mathrm{km} \mathrm{s}^{-1} \mathrm{kpc}^{-1}$ for the GB, which is undoubtedly contaminating their sample.

Some other recent studies find values of $A$ and $B$ in good agreement with ours. Uemura et al. (2000), for a sample of O-B5 Hipparcos stars, estimate that $A=14.0 \pm 0.7 \mathrm{~km} \mathrm{~s}^{-1} \mathrm{kpc}^{-1}$ and $B=-15.8 \pm 0.7 \mathrm{~km} \mathrm{~s}^{-1} \mathrm{kpc}^{-1}$. Zhu (2000), working with O-B5 stars with Hipparcos proper motions, finds that $A=16 \pm 1 \mathrm{~km}$ $\mathrm{s}^{-1} \mathrm{kpc}^{-1}$ and $B=-15.6 \pm 0.8 \mathrm{~km} \mathrm{~s}^{-1} \mathrm{kpc}^{-1}$. Olling \& Dehnen (2003), for a sample of young main-sequence stars, find that $(A$, $B)=(9.6,-11.6) \mathrm{km} \mathrm{s}^{-1} \mathrm{kpc}^{-1}$, while for a sample of red giants, with no significant contribution from the GB, the disk rotation curve is almost flat, $(A, B)=(15.9,-16.9) \mathrm{km} \mathrm{s}^{-1} \mathrm{kpc}^{-1}$, in very good agreement with our results for the OB stars of the LGD.

Our estimation of the circular rotation speed, considering that the Sun's distance to the Galactic center is $8.5 \mathrm{kpc}$ (Kerr \& LyndenBell 1986), is $\Theta_{\odot}=272 \mathrm{~km} \mathrm{~s}^{-1}$ for the LGD from proper motions. It is a high value but in agreement with recent studies of O-B5 star samples from the Hipparcos catalog by Miyamoto \& Zhu (1998), $\Theta_{\odot}=268.7 \pm 11.9 \mathrm{~km} \mathrm{~s}^{-1}$; Uemura et al. (2000), $\Theta_{\odot}=255.52 \pm$ $8.33 \mathrm{~km} \mathrm{~s}^{-1}$; or Branham (2002), $\Theta_{\odot}=258.7 \pm 34.29 \mathrm{~km} \mathrm{~s}^{-1}$. A similar result $\left(\Theta_{\odot}=270 \mathrm{~km} \mathrm{~s}^{-1}\right)$ is also reached by Méndez et al.
(2000) using data from the Southern Proper Motion Catalog (Platais et al. 1998); thus, their result is obtained from measures of the proper motions independent of those from Hipparcos.

\section{CONCLUSIONS}

In Paper I we concluded that in the young Galactic disk there are two spatially different systems, the GB and the LGD. Now we have classified using only spatial criteria a sample of O-B6 stars from the Hipparcos catalog and have found that the GB's global kinematics is essentially different from the kinematics of the LGD. Not only that, multidimensional two-sample tests prove that the GB and the LGD are separated systems in phase space. This does not necessarily imply that the GB is a coherent structure born from a single source such as a giant molecular cloud. We just note that in its present state, the GB is a local system (whose size is well represented by our sample) showing very different kinematic properties than the larger system in which it is embedded, the Galactic disk. The fact that the GB is mainly composed of certain moving groups challenges the idea of this system coming from a single origin, and raises the question of whether we are witnessing a physical entity with coherent structure or are just observing a transitory picture of several smaller systems with no common origin at all. The answer to this question can only be sought in the dynamical study of the moving groups that form the GB (which would require a deep knowledge of the Galactic potential and its asymmetries), in order to trace their trajectories back to the past and discover whether they come from a single protostellar cloud or are the transitory result of some dynamical traps.

We have also proved that the classic problem of the negative vertex deviation of young stars in the solar neighborhood is caused by the contribution of the GB. This effect disappears once this stellar system is removed from the sample, leaving the LGD, defined by its spatial distribution, as the only remaining structure.

Finally, we have observed how the presence of what is called the GB introduces disturbances in the estimation of the Oort constants that describe the kinematics of the young Galactic disk, making it necessary to discard its contribution by identifying and removing the GB members. Once the young disk is pruned by eliminating the GB members, we estimate a flat rotation curve with a local velocity very close to the values calculated by several authors in the last decade for a wide range of ages.

Thus, although we find that a kinematic analysis is not enough to decipher the origin of the GB, it is indeed fundamental to characterizing the complexity of the young Galactic disk and to better understanding the different moving groups that form the bulk of the GB stellar component. A comprehensive explanation of the origin of the GB will require a dynamical analysis of these moving groups.

F. E. wants to thank the Departamento de Física Atómica, Molecular, y Nuclear of the Universidad de Sevilla for its support during this work. We would also like to acknowledge the funding from MCEyD of Spain through grants AYA 2004-05395, and AYA 200408260-C03-02, and from the Consejería de Educación y Ciencia (Junta de Andalucía) through TIC-101.
Arenou, F., \& Luri, X. 1999, in ASP Conf. Ser. 167, Harmonizing Cosmic Distance Scales in a Post-Hipparcos Era, ed. D. Egret \& A. Heck (San Francisco: ASP), 13

Asiain, R., Figueras, F., Torra, J., \& Chen, B. 1999, A\&A, 341, 427

Balona, L. A., \& Shobbrook, R. R. 1984, MNRAS, 211, 375

\section{REFERENCES}

Barbier-Brossat, M., \& Figon, P. 2000, A\&AS, 142, 217

Baringhaus, L., \& Franz, C. 2004, J. Multivariate Anal., 88, 190

Blaauw, A. 1956, ApJ, 123, 408

Bonneau, M. 1964, J. Obs., 47, 251

Branham, R. L., Jr. 2002, ApJ, 570, 190 
Brown, A. G. A. 2002, in ASP Conf. Ser. 285, Modes of Star Formation and the Origin of Field Populations, ed. E. K. Grebel \& W. Brandner (San Francisco: ASP), 150

Cabrera-Caño, J., \& Alfaro, E. J. 1985, A\&A, 150, 298

Chen, B., Asiain, R., Figueras, F., \& Torra, J. 1997, A\&A, 318, 29

Clube, S. V. M. 1972, MNRAS, 159, 289

Comerón, F. 1992, Ph.D. thesis, Univ. Barcelona 1999, A\&A, 351, 506

Comerón, F., \& Torra, J. 1991, A\&A, 241, 57

Comerón, F., Torra, J., \& Gómez, A. E. 1994, A\&A, 286, 789

Dehnen, W. 1998, AJ, 115, 2384

Delhaye, J. 1965, in Galactic Structure, ed. A. Blaauw \& M. Schmidt (Chicago: Univ. Chicago Press), 61

de Zeeuw, P. T., Hoogerwerf, R., de Bruijne, J. H. J., Brown, A. G. A., \& Blaauw, A. 1999, AJ, 117, 354

Eggen, O. J. 1963, AJ, 68, 697 . 1996, AJ, 112, 1595

Elias, F., Cabrera-Caño, J., \& Alfaro, E. 2006, AJ, 131, 2700 (Paper I)

Elmegreen, B. G. 1982, in Submillimeter Wave Astronomy, ed. J. E. Beckman \& J. P. Phillips (Cambridge: Cambridge Univ. Press), 3

Elmegreen, B. G., Efremov, Y., Pudritz, R. E., \& Zinnecker, H. 2000, in Protostars and Planets IV, ed. V. Mannings, A. P. Boss, \& S. S. Russell (Tucson: Univ. Arizona Press), 179

Fernández, D. 2005, Ph.D. thesis, Univ. Barcelona

Filin, A. Ia. 1957, Soviet Astron., 1, 517

Franz, C. 2004, Cramer: Multivariate Nonparametric Cramer Test for the TwoSample Problem (Vienna: R Foundation Stat. Comput.)

Fricke, W., \& Tsioumis, A. 1975, A\&A, 42, 449

Frogel, J. A., \& Stothers, R. 1977, AJ, 82, 890

Gould, B. A. 1879, Result. Obs. Nac. Argentino Cordoba, 1, 354

Grenier, S., et al. 1999, A\&AS, 137, 451

Guillout, P., Sterzik, M. F., Schmitt, J. H. M. M., Motch, C., \& Neuhäuser, R. 1998, A\&A, 337, 113

Hauck, B., \& Mermilliod, M. 1998, A\&AS, 129, 431

Herschel, J. F. W. 1847, Results of Astronomical Observations Made during the Years 1834-1838 at the Cape of Good Hope (London: Smith, Elder, \& Co.)

Johnson, D. R. H., \& Soderblom, D. R. 1987, AJ, 93, 864

Kaltcheva, N., \& Knude, J. 1998, A\&A, 337, 178

Kerr, F. J., \& Lynden-Bell, D. 1986, MNRAS, 221, 1023

Lesh, R. J. 1968, ApJS, 17, 371

Lindblad, P. O. 1967, Bull. Astron. Inst. Netherlands, 19, 34
Maíz-Apellániz, J. 2001, AJ, 121, 2737

2005, in The Three-dimensional Universe with Gaia (ESA SP-576; Noordwijk: ESA), 179

Maíz-Apellániz, J., Walborn, N. R., Galué, H. Á., \& Wei, L. H. 2004, ApJS, 151,103

Méndez, R. A., Platais, I., Girard, T. M., Kozhurina-Platais, V., \& van Altena, W. F. 2000, AJ, 119, 813

Meynet, G., Mermilliod, J.-C., \& Maeder, A. 1993, A\&AS, 98, 477

Mihalas, D., \& Binney, J. 1981, Galactic Astronomy: Structure and Kinematics (San Francisco: Freeman)

Miyamoto, M., \& Zhu, Z. 1998, AJ, 115, 1483

Montes, D., López-Santiago, J., Gálvez, M. C., Fernández-Figueroa, M. J., De Castro, E., \& Cornide, E. 2001, MNRAS, 328, 45

Moreno, E., Alfaro, E. J., \& Franco, J. 1999, ApJ, 522, 276

Olano, C. A. 1982 , A\&A, 112, 195

Olling, R. P., \& Dehnen, W. 2003, ApJ, 599, 275

Perrot, C. A., \& Grenier, I. A. 2003, A\&A, 404, 519

Perryman, M. A. C., et al. 1997, The Hipparcos and Tycho Catalogues (ESA SP-1200; Noordwijk: ESA)

Piskunov, A. E., Kharchenko, N. V., Röser, S., Schilbach, E., \& Scholz, R.-D. 2006, A\&A, 445, 545

Platais, I., et al. 1998, AJ, 116, 2556

Proctor, R. 1869, Proc. R. Soc. London, 18, 169

R Development Core Team 2005, R: A Language and Environment for Statistical Computing (Vienna: R Foundation Stat. Comput.), http://www.R-project.org

Schmidt-Kaler, Th. 1982, in Landolt-Bornstein, Numerical Data and Functional Relationships in Science and Technology, Vol. 2, ed. K. H. Hellwege (Berlin: Springer), 19

Schröder, S. E., Kaper, L., Lamers, H. J. G. L. M., \& Brown, A. G. A. 2004, A\&A, 428, 149

Smart, W. M. 1968, Stellar Kinematics (New York: Wiley)

Stauffer, J. R., Hartmann, L. W., Prosser, C. F., Randich, S. Balachandran, S., Patten, B. M., Simon, T., \& Giampapa, M. 1997, ApJ, 479, 776

Stauffer, J. R., et al. 1999, ApJ, 527, 219

Torra, J., Fernández, D., \& Figueras, F. 2000, A\&A, 359, 82

Turon, C., et al. 1992, The Hipparcos Input Catalogue (ESA SP-1136; Noordwijk: ESA)

Uemura, M., Ohashi, H., Hayakawa, T., Ishida, E., Kato, T., \& Hirata, R. 2000, PASJ, 52, 143

Westin, T. N. G. 1985, A\&AS, 60, 99

Zhu, Z. 2000, PASJ, 52, 1133 